\title{
Eigen Face Vector (EFV) based Personal Assistant through Facial Image Recognition
}

\author{
Roopa JayaSingh J $\mathbf{1}$, Jeba Kumar R.J.S ${ }^{2}$, K. Martin Sagayam ${ }^{3}$, Hanafy M. Ali ${ }^{4}$ \\ ${ }^{1,3}$ Assistant Professor, Department of Electronics and Communication Engineering, Karunya Institute \\ of Technology and Sciences, Coimbatore-641114, India. \\ roopa@karunya.edu, martinsagayam.k@gmail.com \\ ${ }^{2}$ UG Student, Department of Electronics and Communication Engineering, Karunya Institute of \\ Technology and Sciences, Coimbatore-641114, India. \\ jebakumarrjs@gmail.com \\ ${ }^{4}$ Computer and Systems Engineering Department, Faculty of Engineering, Minia University, El \\ Minia, Egypt.
}

Article History: Received: 10 November 2020; Revised 12 January 2021 Accepted: 27 January 2021; Published online: 5 April 2021

\begin{abstract}
This research paper is carried out to solve the issue faced in the busy life of the entire human race to complete all the necessary daily work within a restricted time limit. These compromises in job completion leads to both problems in society and self degradation. This paper talks about the novel low cost personal reminder hub for commons using eigen face vector recognition and reduced Personal Component Analysis (PCA).Personal face recognition, voice reminder which is trained is provided with appropriate reminders to inform us during our busy problems. We train the reminders inorder to find out each person's face average weight parameters which is calculated using cluster matrix calculation. When the recipient of the reminder fixes his face in the face recognistion field, hub acquires the face image and tries to compare wth the captured threshold value to all the prelearnt separate threshold values.From the right match with consideration of minimal deviation the face is lightly recognised and the required saved reminder is informed through the inbuilt voice which would change according to our taste. Thus the efficient method of face identification voice reminder for reduced low cost personal reminder is built and this will be helpful for all busy working people and for family reminders like childrens or working people task reminder, patient tablet reminder etc.
\end{abstract}

Keywords - Face recognition, Personal Component Analysis, Gray Scale, Eigen Vector

\section{INTRODUCTION}

Eigenfaces are the name designated to the group of eigen vectors for Human-Computer contact by minimal face identfication. The difficult task of face identfication inputs the critique input face image which is corrupted but it follows a sequential pattern of a human face, which is identified by scanning the face of the image in the form of many sub-unital(eyes, nose, mouth) facial analysis and the final output is the scanned full facial image. This kind of componental analysis is known as eigen faces in the huge domain of human facial recognistion. Principal Component Analysis (PCA) is a mathematical tool from the extracted real image [1].After Personal Componet Analysis (PCA) was introduced we can change the training images to a set of required eigenfaces, which would display itself as a library source file for face recognition. On marginal weight mapping of the gathered unknown facial image with the trained weight parameter results in the Marginal Deviation value (MD). The captured image is projected in eigen space i.e. grouping of eigen vectors for a linear transformation space. The pretrained system compares the lie feed with the vector comparision which is already stored in its memeory and classifies the live feed as recognised or on-recognised image. The functuional flow of the Eigen Face Vector technique is as depiced in Figure 1. 


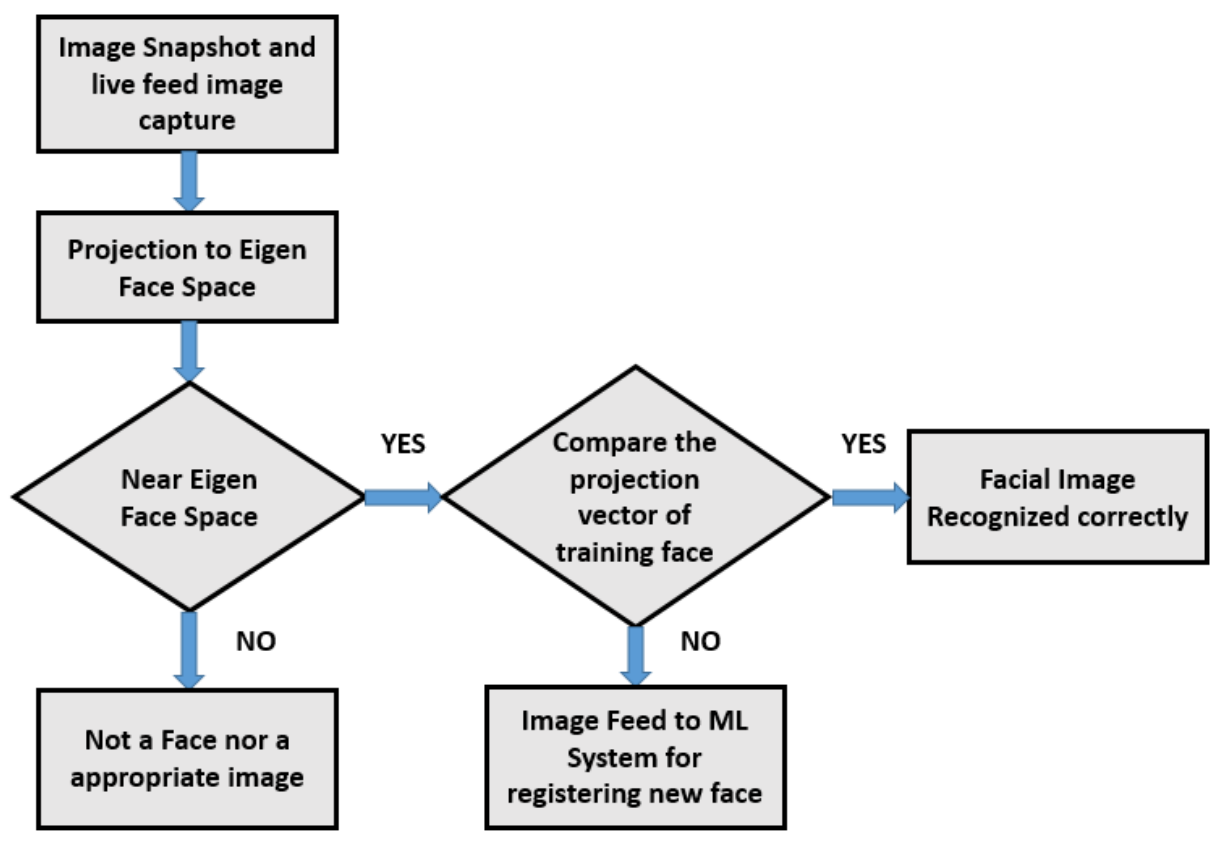

Figure 1. Functional flow chart of Eigen face vector algorithm.

\section{EXISTING WORK}

Currently used personal reminder's used are of android application based holding heavy ram performance mobile which is expensive and also holds heavy compatability problems. Application oriented reminder hugely spoils the entire working capacity of the smart phones due to its consuming memory and clock powering modes even in rest period.

\section{A.Literature Survey}

Prasetyawidi Indrawan et. al. has introduced using android oriented face identificaton which makes the entire system working to be slowed down [2]. Ishwar s Jadhev et. al. has introduced this wide compromising weight methodology, which spoils the performance matrix of face recognization mechanism and thus the full system power of facial mapping is affected [3]. Jasmin Kurti et. al. have also used this concept only with speech identification not with face identification and detection technology and this wont be a real solution to solve the patient problems as the person who is suffering may not be in the place to talk with the module during the serious times [4]. security issues from illegal attackers. For to surpass this issue we introduced the economic facial recognistion voice reminder system as a individual handy module.

\section{PROPOSED SYSTEM}

A novel reminder hub is designed with micro python coding and it is made as a small stand-alone product which can be consumed with a casing and it is made complatable to place it in working peoples table, childrens learning room and also over the patients bed as a self care takers for forecasting the tablets taking time. Security problems are takenover in this project by the friend and the enemy's face by using narrow marginal face compromisation to less than one third of the original compromisation factor.

The separate training faces are to sent initially in the database file and the average weight of the personal component is calculated and it is differentiated with the weight performa of the new face image gathered. When the deviation in the gathered weight matrix and the value of the previously stored weight in the data base is within the reduced deviation the required colour test facial picture is sent to the console for output along with the required presetted voice reminder.The full system is found to be concise with extra feature like benificial human-computer communication. 


\section{A.Identification of Problem and need for this Personal Reminder}

After introduction of rising task to be finished within a required time in this hectic life is the major factor for receiving good reputation in the society. Working people are dumped with multiple tasks to be finished within the required time, hence this prime reminder will be a gift to resolve this problem. Parents to help and plan the children in a proper path to make them to finish their task in required time, this reminder hub is used to monitor students by checking the rude nature of the children have to be helped in all walks of their routine life, it is the major duty of informing them the task to be completed which is decided by parents in the reminder hub before.This low price small product can be used as a live patient pill reminder to inform the systematic and periodic pill taking patients as they may neglect to take their pills which may lead to diasastrous medicinal diseases which may lead to decline of physical health.

\section{B.Functional Flow Process of the proposed Personal Face Identification Voice Reminder (PFIVR)}

The full functional flow of the project result is shown in the Fig. 2, which stresses the facial images of the user are being instructed by the human computer interaction system i.e. Personnal voice reminder hub by the eigen fac vector recognition methodology and it is differentiated with the test faces to get the perfect match

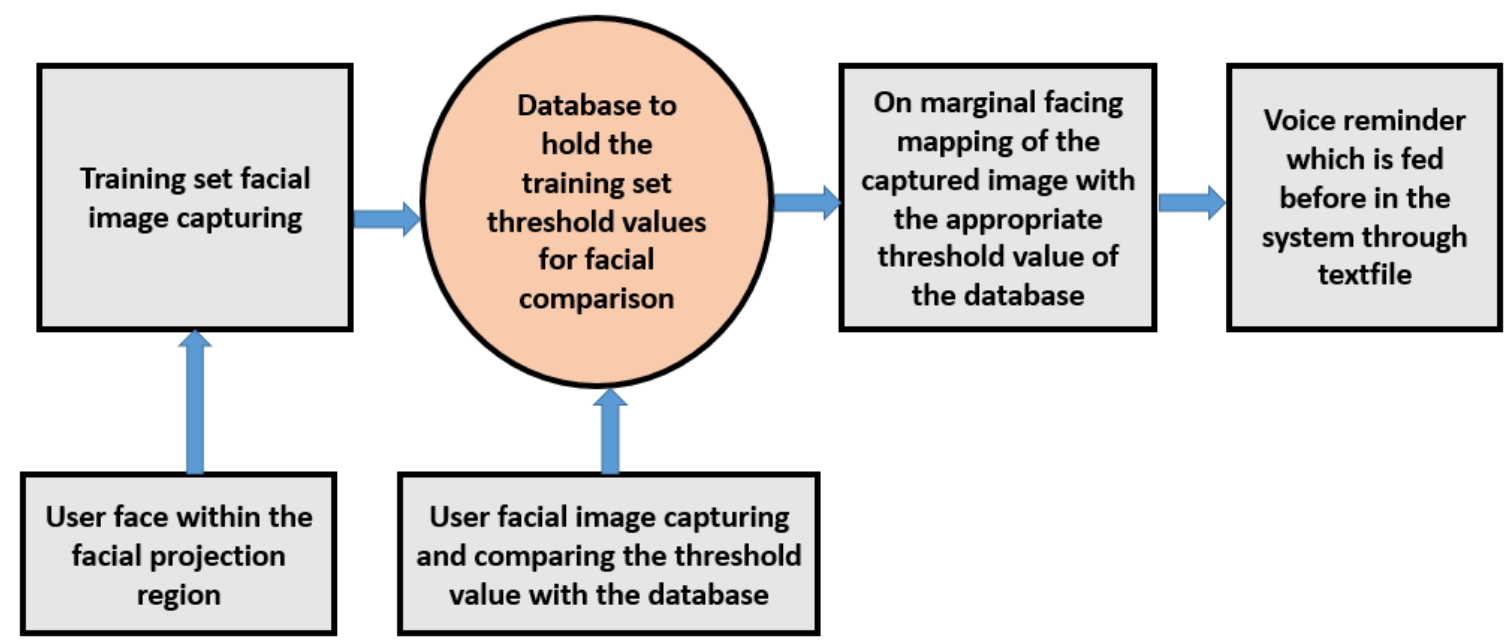

Figure 2. Block Diagram of the Personal Face Identification Voice Reminder (PFIVR).

\section{C.Backend database of the training images}

Training set (Gray Scale facial image database) in the paradigm of face recognistion is the group of alike facial pictures in contrasting data sets to train the recognistion system to find the high resemblance in the face pictures to find the face of the person easily. Training images are captured before the system operation for to find the correct person with required lightings to reduce the mistakes [5],[6]. Training the images at the maximum limit of 25 images to train the module of face for patient data base is as shown below in Fig. 3 . 


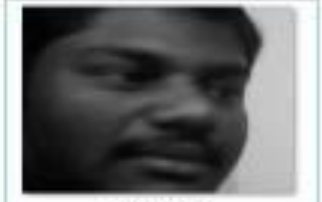

10.jpg

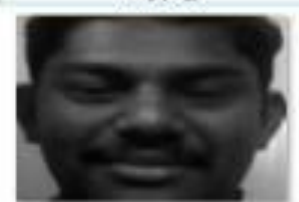

22.jpg

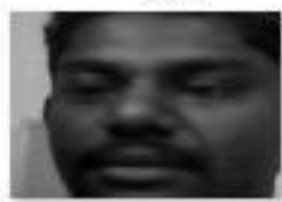

16.jpg

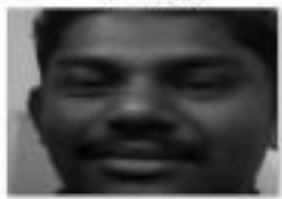

19.jpg

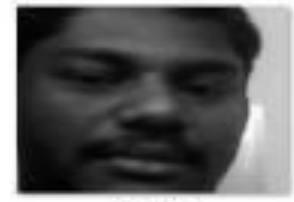

14.jpg

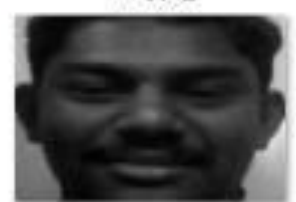

23.jpg

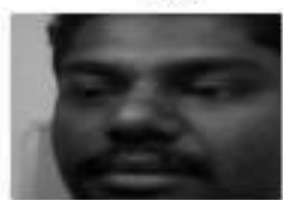

17.jpg

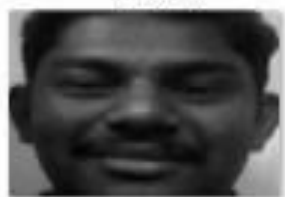

20.jpg

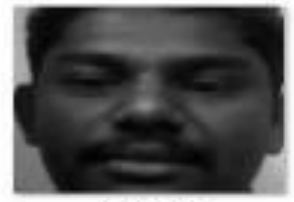

15.jpg

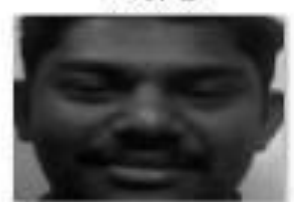

24.jpg

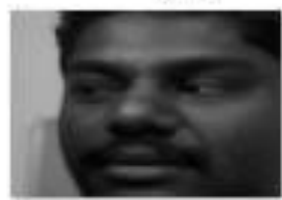

18.jpg

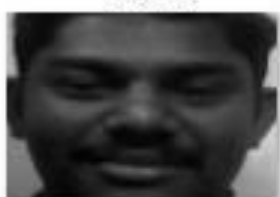

24.jpg

Figure. 3. Training images for patient database to calculate the weight of face for the particular patient

Care has to be taken for the facial projection in the optimal PCA sale of projection to reduce the harsh facial recognition and to have required lighting effect to reduce the shadow effect of the gray scale images. Training facial sample images for student database is as depicted in Fig. 4.

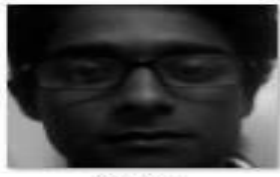

9.jpg

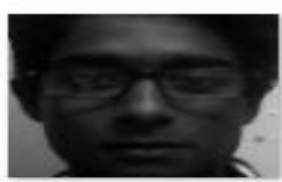

17.jpg

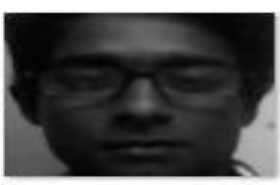

12.jpg

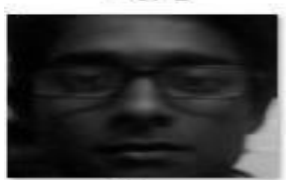

21.jpg

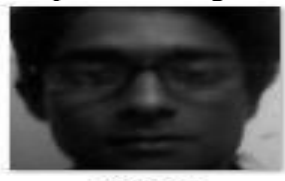

10.jpg

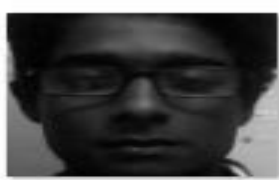

18.jpg

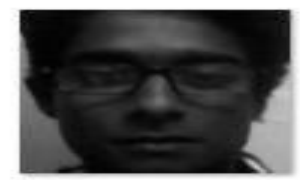

13.jpg

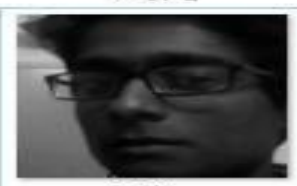

23.jpg

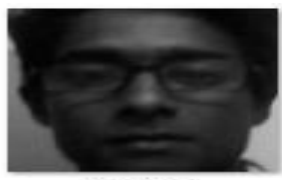

11.jpg

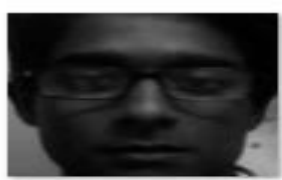

19.jpg

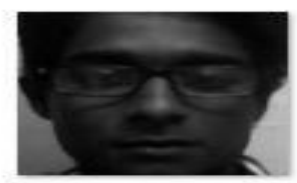

14.jpg

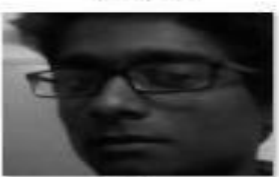

24.jpg

Figure. 4. Training images for student database to find the facial weight of the particular student

\section{D.Mathematical Equation for Eigen Vector face recognition}

Eigen vector procedures and its functional technlogies are executed for the facial vector analysis [7]-[9]. Here we take the training faces Fi of size $\mathrm{m}$ by $\mathrm{n}$. The image matrix of $\mathrm{m}$ by $\mathrm{n}$ is changed into a vector of size $\mathrm{x}$ by $\mathrm{y}$. The training faces of the faces is provided as T1, T2, etc. and an average A is calculated by the equation 


$$
\mathrm{A}=\frac{1}{\mathrm{~N}} \sum_{i=1}^{N} T i
$$

The vector $Y_{i}=T_{i}-A$ is the contrast image for indvdual image

faces and it is normalised as one quantity as a resultant weight Wi.This training of face is done for all person by projecting their faces in the proper projection axis.

Covariance matrix $(\mathrm{Ci})$ of the each facial image $T i$ is given as

$$
\mathrm{C}=\frac{1}{\mathrm{~N}} \sum_{i=1}^{N} T i T_{i}^{T}
$$

Relation between eigen vector $\mathrm{Vi}$ and eigen values $\chi_{\mathrm{i}}$ is given by

$$
\mathrm{CV}_{\mathrm{i}}=\chi_{\mathrm{i}} \mathrm{V}_{\mathrm{i}}
$$

The minimal distance between the test face $T_{f}$ and training face $T i$ is given as Euclidian distance $\left(E_{d}\right)$

$$
E_{d}=\sqrt[2]{\left|\left(T_{f}-T_{i}\right)\right|^{2}}
$$

The Euclidian distance $\left(E_{d}\right)$ inputs values are inputted between 0 and 150 for the top facial match condition i.e $0<E_{d}<150$. The recognized face weight lies within the range of optimal Euclidian distance [10]. Main goal of this project is to provide the voice output and this is gained by the Python text to speech conversion i.e PYTTSX, a cross platform for text to speech engine. Fig. 5., shows the result of face recognised voice reminder for the required patient from the previous stored database. Fig. 6., shows the output of face recognised voice reminder for the required student from the previous saved database.

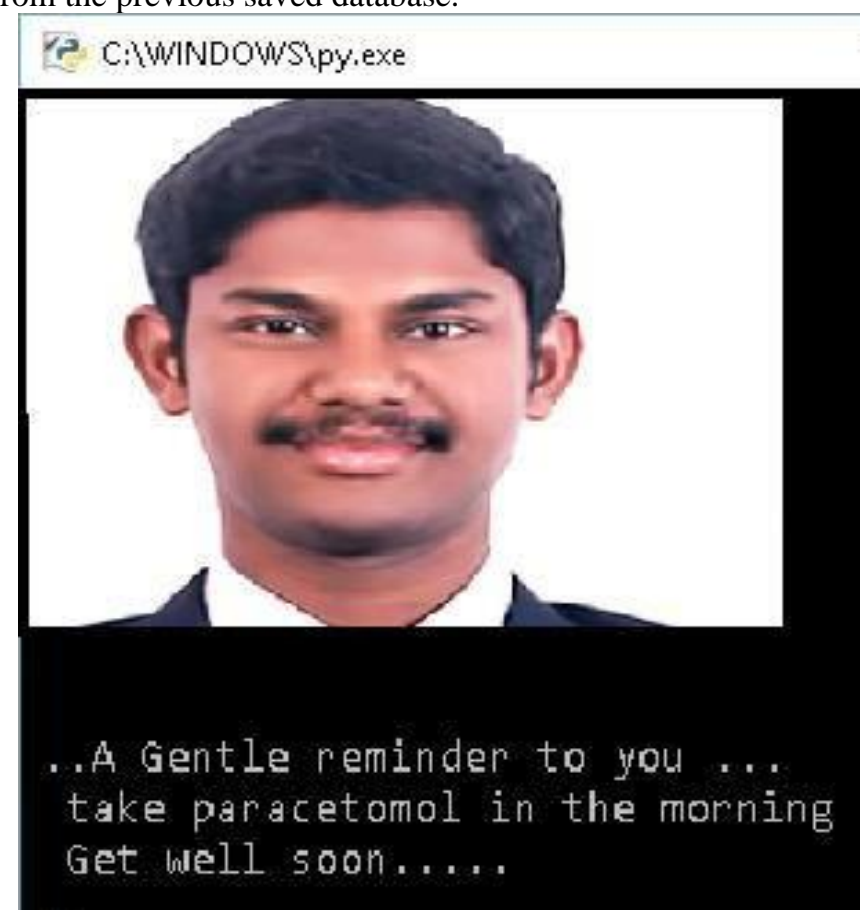

Figure. 5. GUI output which depicts the reminder to the identfied patient together with the voice reminder 


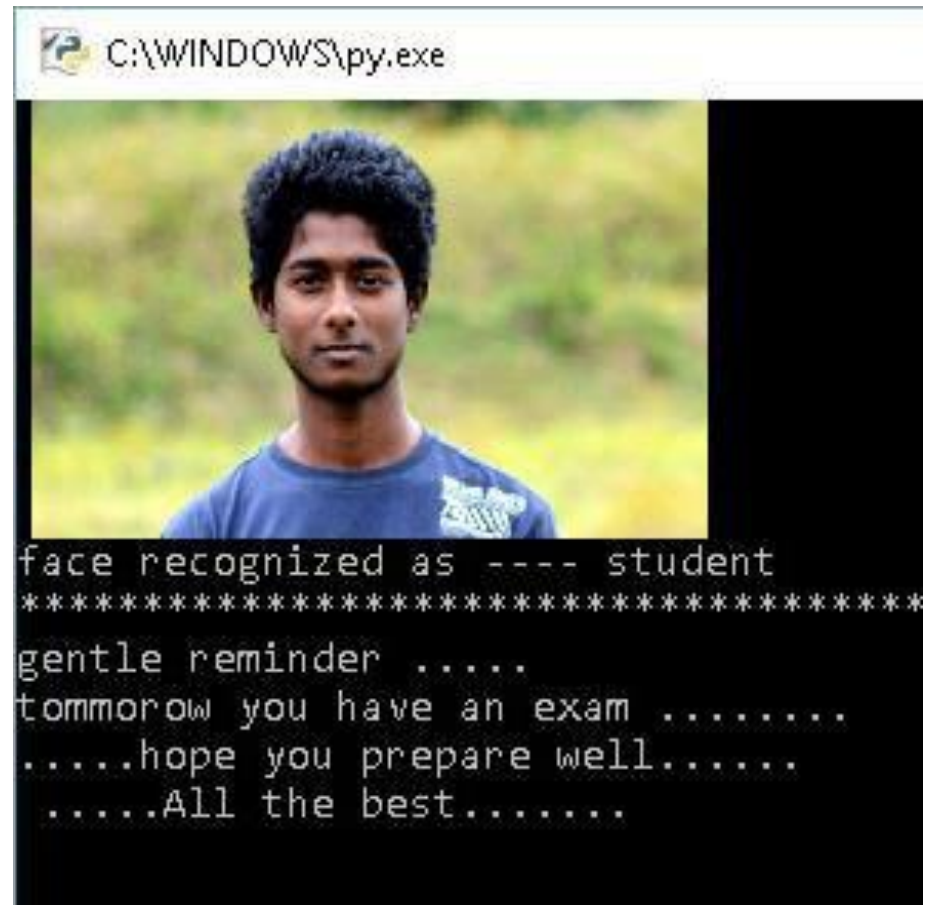

Figure. 6. GUI output which depicts the student reminder to the recognized facial image

\section{CONCLUSION}

This research paper showcases the good user friendly face recognition reminder hub, which is a huge gift to all working people, student community for machine intimated parental care and especially for older people as a early reminder to take the pills at periodic times. The integration of Eigen Face Vector based recognition helps to achive the efficient facial image recognition in a simple and direct technique fo comparing the caprtured image with eigen space of projection. This research paper also gives the flexibility of addition of facial image with the help of Machine Learning (ML)This project work will be developed in future by introducing the networking fuzzy logic for data prediction and artificial intelligence technology thereby to make it a still more solvable solution to resolve the problems humanity faces everyday and to make life at ease with automatic technological reminder.

\section{REFERENCES}

[1] Singh, G., \& Goel, A. K. (2020, March). Face Detection and Recognition System using Digital Image Processing. In 2020 2nd International Conference on Innovative Mechanisms for Industry Applications (ICIMIA) (pp. 348-352). IEEE.

[2] Rana, A., \& Hajati, F. (2020, April). A Survey on Privacy and Security in Mobile Cloud Computing. In Workshops of the International Conference on Advanced Information Networking and Applications (pp. 1065-1076). Springer, Cham.

[3] Kuśmierczyk, A., Sławińska, M., Żaba, K., \& Saeed, K. (2020). Biometric Fusion System Using Face and Voice Recognition. In Advanced Computing and Systems for Security (pp. 71-89). Springer, Singapore.

[4] Isyanto, H., Arifin, A. S., \& Suryanegara, M. (2020, October). Performance of Smart Personal Assistant Applications Based on Speech Recognition Technology using IoT-based Voice Commands. In 2020 International Conference on Information and Communication Technology Convergence (ICTC) (pp. 640645). IEEE.

[5] Dawson, J., Goodwyn, J., Means, S., \& Crakes, J. (2020). Quality and Match Performance Analysis of BandFiltered Visible RGB Images. In Securing Social Identity in Mobile Platforms (pp. 105-128). Springer, Cham.

[6] Oloyede, M. O., Hancke, G. P., \& Myburgh, H. C. (2020). A review on face recognition systems: recent approaches and challenges. Multimedia Tools and Applications, 79(37), 27891-27922.

[7] Titijaroonroj, T., Hancherngchai, K., \& Rungrattanaubol, J. Regional Covariance Matrix-Based TwoDimensional PCA for Face Recognition. In 2020 12th International Conference on Knowledge and Smart Technology (KST) (pp. 6-11). IEEE. 
[8] Chen, L. F., Liao, H. Y. M., Ko, M. T., Lin, J. C., \& Yu, G. J. (2000). A new LDA-based face recognition system which can solve the small sample size problem. Pattern recognition, 33(10), 1713-1726.

[9] Latha, P., Ganesan, L., \& Annadurai, S. (2009). Face recognition using neural networks. Signal Processing: An International Journal (SPIJ), 3(5), 153-160.

[10] Majumdar, S., Bose, A., \& Das, P. (2021). A Novel Approach for Face Recognition Using Modular PCA and MAP-MRF Classifier. In Proceedings of International Conference on Frontiers in Computing and Systems (pp. 181-189). Springer, Singapore. 